\title{
Mechanochemically Synthesised Dicyanamide Hybrid Organic- Inorganic Perovskites and their Melt-Quenched Glasses
}

Received 00th January 20xx, Accepted 00th January 20xx DOI: $10.1039 / x 0 \times x 00000 x$

\author{
Lauren N. McHugh, ${ }^{a}$ Michael F. Thorne, ${ }^{a}$ Ashleigh M. Chester, ${ }^{a}$ Martin Etter, ${ }^{b}$ Krunoslav Užarević, \\ and Thomas D. Bennett*a
}

Here we present efficient and scalable mechanochemical formation of hybrid organic-inorganic perovskites of the form $[\operatorname{TPA}]\left[\mathrm{M}(\mathrm{dca})_{3}\right]\left(\mathrm{M}=\mathrm{Mn}^{2+}, \mathrm{Co}^{2+}\right)$ and the subsequent formation of their bulk melt-quenched glass samples. The thermal, chemical and adsorptive properties of the materials are also investigated.

Hybrid organic-inorganic perovskites (HOIPs) with the formula $\mathrm{ABX}_{3}(\mathrm{~A}=$ organic cation, $\mathrm{B}=$ metal cation and $\mathrm{X}=$ anion $)$ are highly important materials within the field of materials science and display characteristics from both metal-organic frameworks (MOFs) and the parent perovskite family. ${ }^{[1]} \mathrm{HOIPs}$ have attracted considerable attention due to their multiferroic properties, ${ }^{[2]}$ and their use as semiconductors ${ }^{[3]}$ and photovoltaics. ${ }^{[4,5]}$ The inclusion of anionic bridging ligands on the X-site such as formate [HCOO-],[6] hypophosphite $\left[\mathrm{H}_{2} \mathrm{PO}_{2}^{-}\right],{ }^{[7]}$ or dicyanamide (dca, $\left[\mathrm{C}_{2} \mathrm{~N}_{3}^{-}\right]$), ${ }^{[8,9]}$ further expand the structural diversity and functionality of this family.

In addition to the previously reported melting of 2D-layered HOIP series such as $\left[(B A)_{2} \mathrm{MI}_{4}\right]\left(B A=\right.$ butylammonium $\left[\mathrm{C}_{4} \mathrm{H}_{12} \mathrm{~N}^{+}\right]$, $\left.\mathrm{M}=\mathrm{Ge}^{2+}, \mathrm{Sn}^{2+}, \mathrm{Pb}^{2+}\right)^{[10]}$ and $\left[(\mathrm{AA})_{2} \mathrm{Pbl}_{4}\right] \quad(\mathrm{AA}=$ alkylammonium), ${ }^{[11]}$ HOIPs with composition [TPrA] [[M(dca $\left.)_{3}\right]$ (TPrA = tetrapropylammonium $\left[\mathrm{C}_{12} \mathrm{H}_{28} \mathrm{~N}^{+}\right], \mathrm{M}=\mathrm{Mn}^{2+}, \mathrm{Co}^{2+}$ and $\mathrm{Fe}^{2+}$ ) were very recently shown to melt at $\mathrm{ca}$. $250^{\circ} \mathrm{C}$. Upon cooling from the liquid state, glass analogues were produced, which displayed interesting thermal and electrical properties. ${ }^{[12]}$ These, along with glasses formed from zeolitic imidazolate frameworks (ZIFs), both belong to the hybrid glass family, though the latter melt at higher temperatures of $c a$. $400{ }^{\circ} \mathrm{C}$. ${ }^{[13]}$ These latter species have shown interesting optical and mechanical properties, ${ }^{[14,15]}$ and display potential in

\footnotetext{
a. Department of Materials Science and Metallurgy, University of Cambridge, 27 Charles Babbage Road, Cambridge, Cambridgeshire, CB3 OFS.

b. Deutsches Elektronen Synchrotron, FS-PETRA-D, P02.1, Notkestr. 85, 22607

Hamburg, Germany.

Ruđer Bošković Institute, Zagreb, Croatia.

E-mail:tdb35@cam.ac.uk

+ Electronic Supplementary Information (ESI) available. See

DOI: 10.1039/x0xx00000x
}

applications such as gas separation and battery technology. ${ }^{[16,17]}$

Mechanochemical synthesis involves solvent-free grinding to induce a chemical reaction and has been utilised for the green and efficient formation of several families of hybrid materials, mostly coordination polymers and MOFs. ${ }^{[18,19]}$ Until now, only the most conventional HOIPs with composition $\mathrm{MAPbl}_{3}\left(\mathrm{MA}=\right.$ methylammonium $\left.\left[\mathrm{CH}_{6} \mathrm{~N}^{+}\right]\right)$and similar mixed cation species with the general formula $\left[(\mathrm{MA})_{x}(\mathrm{FA})_{1-\mathrm{x}} \mathrm{Pbl} \mathrm{l}_{3}\right],(\mathrm{FA}=$ formamidinium $\left.\left[\mathrm{CH}_{5} \mathrm{~N}_{2}^{+}\right]\right)$have been synthesised via mechanochemistry. ${ }^{[20,21]}$

HOIPs are predominantly prepared by slow crystallisation from layered solutions over several weeks. ${ }^{[8,9]}$ Such techniques are somewhat inefficient and commonly rely on the use of highvapour-pressure organic solvents, resulting in energyconsuming work-ups and the production of organic solvent waste. This long and costly processing, in turn, impacts the availability and potential cost of HOIP-based materials, such as their glasses, particularly on larger scales. The lower melting temperatures $\left(T_{\mathrm{m}} \mathrm{s}\right)$ of HOIPs mean that processing into the bulk scale is, in theory, easier. Therefore, routes to increase the efficiency of and to scale-up the synthesis of the crystalline precursors are actively sought.

Here we present the first mechanochemical synthesis of HOIPs of the form [TPrA] [[M(dca $\left.)_{3}\right]$ as a rapid, green and efficient method for producing perovskite materials and the subsequent formation of their melt-quenched glasses on a larger scale. Two members of the $[\operatorname{TPrA}]\left[\mathrm{M}(\mathrm{dca})_{3}\right]$ family of HOIPs: [TPrA] $\left[\mathrm{Mn}(\mathrm{dca})_{3}\right],{ }^{[8]}$ and $[\operatorname{TPrA}]\left[\mathrm{Co}(\mathrm{dca})_{3}\right]^{\left[{ }^{[9]}\right.}$ were studied in this work. [TPrA] $\left[\mathrm{Mn}(\mathrm{dca})_{3}\right]$ forms in the tetragonal space group $P \overline{4} 2_{1} c$, while [TPrA] [Co $(\mathrm{dca})_{3}$ ] forms in the orthorhombic space group Pnna. Both structures contain bridging dca ligands, which bind through the $\mathrm{N}$ atoms to the transition metal cations (Figure 1a).

In a typical mechanochemical experiment, crystalline HOIP samples were formed after 30 minutes of milling (see methods), where the quantity of the liquid additive was optimised for the synthesis of [TPrA] $\left[\mathrm{Mn}(\mathrm{dca})_{3}\right]$ (Figure S1). The reaction was 


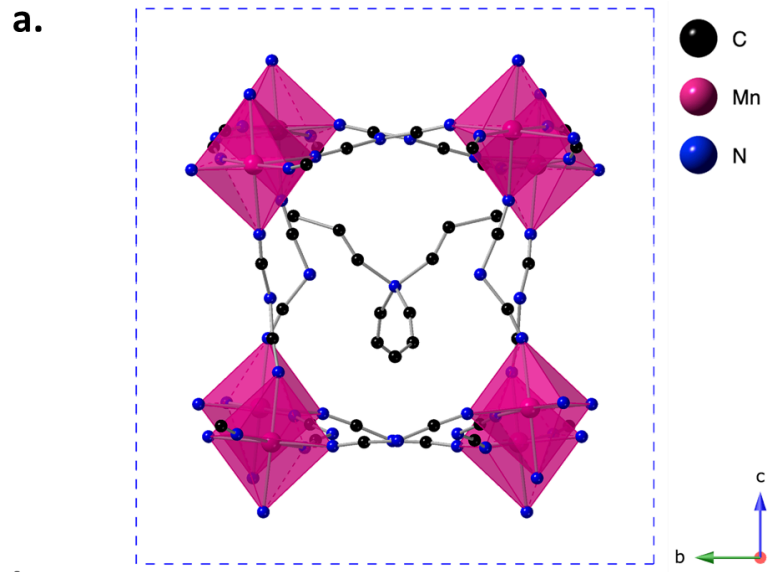

b.

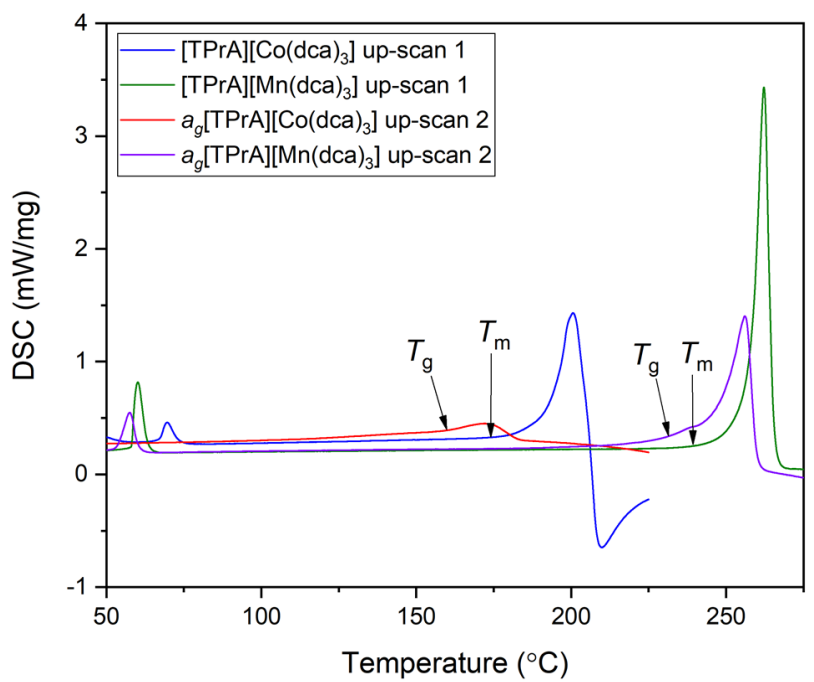

Figure 1a. Simplified structure of $[\operatorname{TPrA}]\left[\mathrm{Mn}(\mathrm{dca})_{3}\right]$, showing one of the possible orientations within the average crystal structure. ${ }^{87} \mathrm{H}$ atoms have been omitted for clarity and only the TPrA ion located on the body-centre position is visible. $b$. DSC traces of $[\operatorname{TPrA}]\left[\mathrm{Co}(\mathrm{dca})_{3}\right]$ and $[\operatorname{TPrA}]\left[\mathrm{Mn}(\mathrm{dca})_{3}\right]$,
showing the first and second up-scans recorded at a heating rate of showing the first and second up-scans recorded at a heating rate of
$10^{\circ} \mathrm{C} \mathrm{min}^{-1}$. The $T_{\mathrm{m}}$ and $T_{\mathrm{g}}$ are shown for each sample and $a_{\mathrm{g}}$ denotes the $10^{\circ} \mathrm{C} \mathrm{min}^{-1}$. The $T_{\mathrm{m}}$ and $T_{\mathrm{g}}$ are shown for each sample and $a_{\mathrm{g}}$ denotes the
glass-phase. A second melting peak is observed close to the $T_{\mathrm{g}}$ for $a_{g}[\mathrm{TPrA}]\left[\mathrm{Mn}(\mathrm{dca})_{3}\right]$ due to significant recrystallisation within the sample upon cooling. The endothermic features at ca. $50-70{ }^{\circ} \mathrm{C}$ represent previously

successfully scaled-up by $5 x$, limited only by the size of milling jar. Samples were initially formed containing sodium chloride and sodium bromide salt by-products (Figure S2, S3), which were readily removed by washing with ice-cold distilled water (Figures S4 and S5). The mechanochemical formation of both [TPrA] $\left[\mathrm{Mn}(\mathrm{dca})_{3}\right]$ and [TPrA] [Co(dca $\left.)_{3}\right]$ was monitored using insitu synchrotron PXRD (see methods). [22] [TPrA][Mn(dca) ${ }_{3}$ ] forms directly within 10 minutes (Figure S6), whereas $[\operatorname{TPr}]\left[\mathrm{Co}(\mathrm{dca})_{3}\right]$ formed almost immediately, in under 1 minute of milling (Figure S7). These facile and efficient, laboratory-scale mechanosyntheses demonstrate the potential for further scaleup, or continuous synthesis using extrusion. ${ }^{[23]}$

Thermogravimetric analysis (TGA) was performed under both argon (Ar) and nitrogen $\left(\mathrm{N}_{2}\right)$ on [TPrA] $\left[\mathrm{Mn}(\mathrm{dca})_{3}\right]$ (Figure S8, S9) and $[\mathrm{TPrA}]\left[\mathrm{Co}(\mathrm{dca})_{3}\right]$ (Figure S10, S11) prior to differential scanning calorimetry (DSC) measurements (Figure 1b), where the two samples were heated, cooled and heated again under $\mathrm{Ar}$, using a heating/cooling rate of $10^{\circ} \mathrm{C} \mathrm{min}-1$.
Significant recrystallisation was observed in the case of $[\operatorname{TPrA}]\left[\mathrm{Mn}(\mathrm{dca})_{3}\right]$ (Figure S12) and the glass transition temperature $\left(T_{\mathrm{g}}\right)$ was situated close to the second melting peak. This is likely due to the sample beginning to decompose immediately after melting. [TPrA] $\left[\mathrm{Mn}(\mathrm{dca})_{3}\right]$ was then cooled at $3{ }^{\circ} \mathrm{C} \mathrm{min-1}$ (Figure $\mathbf{S 1 3}$ ) in an attempt to reduce the extent of recrystallisation upon cooling, which was ultimately successful. The sample showed little to no recrystallisation on the cooling step, and a $T_{\mathrm{g}}$ was again evident. Comparable DSC analysis was performed on [TPrA] $\left[\mathrm{Co}(\mathrm{dca})_{3}\right]$, where one sample was heated, cooled and heated at a rate of $10{ }^{\circ} \mathrm{C} \mathrm{min}^{-1}$ (Figure S14) and another where $[\mathrm{TPrA}]\left[\mathrm{Co}(\mathrm{dca})_{3}\right]$ was cooled at $3^{\circ} \mathrm{C} \mathrm{min}^{-1}$ (Figure S15). Cooling at a slower rate again reduced the extent of recrystallisation in the sample. A $T_{\mathrm{g}}$ was observed for the sample cooled at $10{ }^{\circ} \mathrm{C} \mathrm{min}-1$, though not for the sample cooled at $3{ }^{\circ} \mathrm{C} \mathrm{min}-1$. The mechanochemically synthesised samples both melted at lower temperatures than samples produced by slow crystallisation (Table S1), likely due to the significantly lower particle size of mechanochemically produced samples. Similar behaviour has been observed in nanoscale materials. [24] Similarly, mechanochemically synthesised samples displayed lower decomposition temperatures $\left(T_{\mathrm{d}} \mathrm{S}\right)$ than traditionally synthesised HOIPs (Table S1). The $T_{\mathrm{g}} \mathrm{s}$ for both samples were higher than those reported in the literature (Table S1). Lowtemperature DSC was also performed under $\mathrm{N}_{2}$ (Figure S16, S17), where samples were heated to the desired temperature, cooled to $-60^{\circ} \mathrm{C}$, then heated again. Both samples displayed broadly comparable $T_{\mathrm{m}}$ and $T_{\mathrm{g}} \mathrm{s}$ to those determined under $\mathrm{Ar}$ (Table S1).

HOIP glass samples produced by Shaw et al.[12] were formed on a small-scale inside an SDT (simultaneous TGA-DSC) furnace, which limited batches to $c a .15 \mathrm{mg}$. SDT was also used here to investigate the optimum temperature and heating/cooling rate to minimise recrystallisation in samples, where samples were heated to close to their $T_{\mathrm{d}}$ to minimise recrystallisation. The samples were heated at $10{ }^{\circ} \mathrm{C} \mathrm{min}^{-1}$, before being cooled at either $10{ }^{\circ} \mathrm{C} \mathrm{min}-1$ or $3{ }^{\circ} \mathrm{C} \mathrm{min}^{-1}$. In agreement with the previous DSC analysis, considerable recrystallisation was observed upon cooling at $10^{\circ} \mathrm{C} \mathrm{min}^{-1}$, for $a_{\mathrm{g}}[\mathrm{TPrA}]\left[\mathrm{Mn}(\mathrm{dca})_{3}\right]$, while cooling at $3{ }^{\circ} \mathrm{C} \mathrm{min}^{-1}$ lead to a fully amorphous $a_{\mathrm{g}}[\mathrm{TPrA}]\left[\mathrm{Mn}(\mathrm{dca})_{3}\right]$ sample (Figure S18). No recrystallisation to the original phase was observed for $a_{\mathrm{g}}[\mathrm{TPrA}]\left[\mathrm{Co}(\mathrm{dca})_{3}\right]$ upon cooling at $10{ }^{\circ} \mathrm{C} \mathrm{min}^{-1}$ or the slower rate of $3^{\circ} \mathrm{C} \mathrm{min}^{-1}$, regardless of the cooling rate (Figure S19). However, some extremely minor Bragg peaks, attributed to impurities, were observed in the sample cooled at $3{ }^{\circ} \mathrm{C} \mathrm{min}^{-1}$.

To produce bulk glass samples, crystalline HOIPs were heated to $260{ }^{\circ} \mathrm{C}$ for $[\operatorname{TPrA}]\left[\mathrm{Mn}(\mathrm{dca})_{3}\right]$ and $240{ }^{\circ} \mathrm{C}$ for $[\operatorname{TPrA}]\left[\mathrm{Co}(\mathrm{dca})_{3}\right]$ within a tube furnace and under flowing Ar. The sample was then cooled to room temperature naturally over several hours. Very minor oxidation was evident in both glass samples at $c a .45^{\circ}$, though no recrystallisation was observed for either (Figure 2). From the previous analysis, it appeared that slow cooling of samples, especially $[\operatorname{TPr}]\left[\mathrm{Mn}(\mathrm{dca})_{3}\right]$, helped limit recrystallisation. This is somewhat contradictory to traditional glasses, where rapid cooling of the melt is typically required for glass formation. [25] 


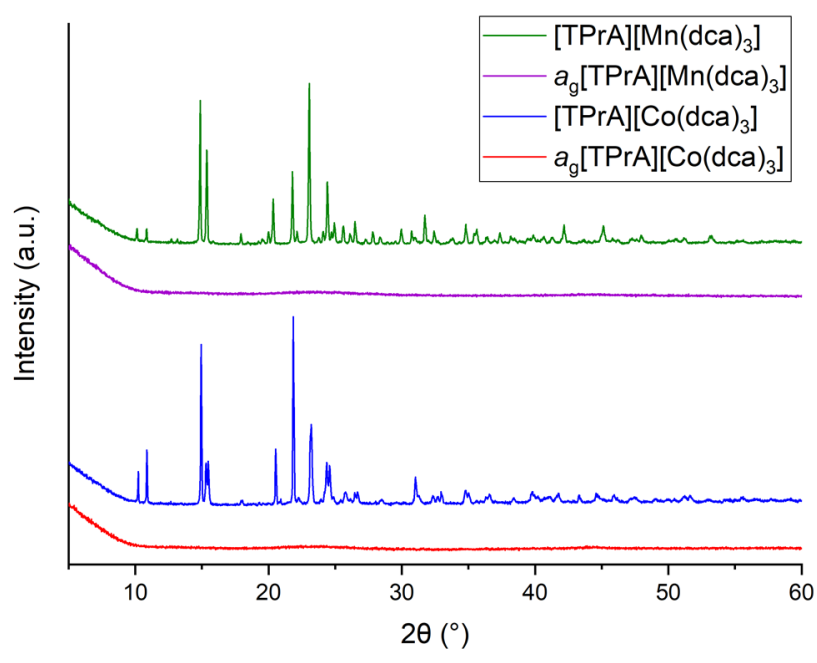

Figure 2. PXRD patterns of both the crystalline and bulk glass forms of [TPrA] $\left[\mathrm{Mn}(\mathrm{dca})_{3}\right]$ and $[\mathrm{TPrA}]\left[\mathrm{Co}(\mathrm{dca})_{3}\right]$.

In glasses derived from HOIPs, there appears to be a complex interplay between glass-formation, decomposition and recrystallisation. With slower cooling, HOIP samples are held at close to their $T_{\mathrm{d}}$ for extended periods, which leads to minor sample decomposition and reduced likelihood of a return to the original, ordered crystalline state.

The initial crystalline sample $[\operatorname{TPrA}]\left[\mathrm{Mn}(\mathrm{dca})_{3}\right]$ consisted of block-like crystals (Figure 20a, b), which appeared to flow together upon glass-formation to give a material with clear surface porosity (Figure S20c, d). Similarly, crystalline [TPrA] $\left[\mathrm{Co}(\mathrm{dca})_{3}\right]$ also consisted of rounded 'blocky' crystals (Figure S21a, b). The sample also appeared to have undergone significant flow upon glass-formation, to provide a relatively smooth material with minor surface porosity (Figure S21c, d).

Crystalline and glass samples of $[\operatorname{TPrA}]\left[\mathrm{Mn}(\mathrm{dca})_{3}\right]$ and $[\operatorname{TPr}]\left[\mathrm{Co}(\mathrm{dca})_{3}\right]$ were exposed to acidic, neutral or basic conditions (see methods) to assess the effect of $\mathrm{pH}$ on the chemical stability of HOIPs and their glasses. Similar studies have been performed both on inorganic glasses and MOF glasses to determine their stabilities. ${ }^{[26,27]} a_{\mathrm{g}}[\mathrm{TPrA}]\left[\mathrm{Mn}(\mathrm{dca})_{3}\right]$ was stable in solutions with $\mathrm{pH} \mathrm{2,5,7}$ and 10, with no recrystallisation to the original crystalline phase, though very minor impurity peaks were present (Figure S22). Like $a_{\mathrm{g}}[\operatorname{TPrA}]\left[\mathrm{Mn}(\mathrm{dca})_{3}\right], a_{\mathrm{g}}[\operatorname{TPrA}]\left[\mathrm{Co}(\mathrm{dca})_{3}\right]$ was also stable in strongly acidic, mildly acidic, neutral and mildly basic solutions, with no evidence of recrystallisation (Figure S23). Most of the crystalline samples dissolved in solutions regardless of $\mathrm{pH}$, though as with glasses, crystalline samples of unknown origin were recovered solely from strongly basic solutions $(\mathrm{pH} 13$, Figure S24, S25). These differences in stability reflect the different local structure present in the crystalline and glass samples.

Pellets of both crystalline and glass samples were synthesised (see methods) (Figures S26 and S27). Optical microscope images of the two glass pellets highlighted the significant differences in the form of the materials upon heating, where $a_{\mathrm{g}}[\mathrm{TPrA}]\left[\mathrm{Co}(\mathrm{dca})_{3}\right]$ formed as a mostly flat pellet with an almost pearlescent appearance (Figure S28a). The
$a_{\mathrm{g}}[\mathrm{TPrA}]\left[\mathrm{Mn}(\mathrm{dca})_{3}\right]$ glass pellet, however, expanded vertically to form a stratified sample with surface cracks (Figure S28b).

The hydrophobicity of pelletised $a_{\mathrm{g}}[\operatorname{TPrA}]\left[\mathrm{Co}(\mathrm{dca})_{3}\right]$ was investigated using water contact angle measurements (see methods, Figure S29). The glass surface was found to be fairly hydrophilic, with a mean water contact angle of $57.53 \pm 1.38^{\circ}$. Measurements were not attempted on $a_{\mathrm{g}}[\operatorname{TPrA}]\left[\mathrm{Mn}(\mathrm{dca})_{3}\right]$ due to the very fragile pellet, which fragmented upon handling.

HOIPs are typically non-porous materials and consequently, gas adsorption is not normally performed. It was proposed by Shaw et al. that the melting mechanism in $[\operatorname{TPrA}]\left[\mathrm{M}(\mathrm{dca})_{3}\right]$ HOIPs involved the movement of the TPrA cation during the melting process, leading to a large structural change. Gas adsorption measurements were subsequently performed to investigate whether this change in conformation upon meltquenching may introduce a degree of porosity into the samples. It is known from work on MOFs that $\mathrm{N}_{2}$ gas cannot enter micropores with a width of less than $4.5 \AA$ due to kinetic restrictions, ${ }^{[28]}$ and as HOIPs are usually non-porous, the smaller kinetic diameter $\mathrm{CO}_{2}$ molecule was used as an analyte in this study to maximise the likelihood of adsorption. The $\mathrm{CO}_{2}$ adsorption isotherms for both the crystalline and glass forms of $[\operatorname{TPrA}]\left[\mathrm{Mn}(\mathrm{dca})_{3}\right]$ and $[\operatorname{TPrA}]\left[\mathrm{Co}(\mathrm{dca})_{3}\right]$ are shown in Figures 3a and $\mathbf{3} \mathbf{b}$ respectively, where $\mathrm{CO}_{2}$ uptakes were recorded up to a relative pressure of $0.035 \mathrm{P} / \mathrm{P}_{0} . \quad[\operatorname{TPrA}]\left[\mathrm{Mn}(\mathrm{dca})_{3}\right]$ and [TPrA] [Co $(\mathrm{dca})_{3}$ ] provided $\mathrm{CO}_{2}$ uptake values of $3.44 \mathrm{~cm}^{3} \mathrm{~g}^{-1}$ and $3.22 \mathrm{~cm}^{3} \mathrm{~g}^{-1}$ respectively, where the very low levels of adsorption highlighted the non-porous nature of the crystalline HOIPs. $a_{\mathrm{g}}[\mathrm{TPrA}]\left[\mathrm{Mn}(\mathrm{dca})_{3}\right]$ and $a_{\mathrm{g}}[\mathrm{TPrA}]\left[\mathrm{Co}(\mathrm{dca})_{3}\right]$ provided $\mathrm{CO}_{2}$ uptake values of $14.90 \mathrm{~cm}^{3} \mathrm{~g}^{-1}$ and $9.00 \mathrm{~cm}^{3} \mathrm{~g}^{-1}$ respectively, and though they are still not considered particularly porous, the $\mathrm{CO}_{2}$ capacities were considerably higher than their crystalline counterparts. This is especially true for $a_{\mathrm{g}}[\operatorname{TPr} \mathrm{A}]\left[\mathrm{Mn}(\mathrm{dca})_{3}\right]$, where the $\mathrm{CO}_{2}$ uptake upon glass formation increased by a factor of 4 . The shapes of the isotherms also changed upon glass formation. Whereas the change was minor for $a_{\mathrm{g}}[\operatorname{TPrA}]\left[\mathrm{Co}(\mathrm{dca})_{3}\right]$, the isotherm of $a_{\mathrm{g}}[\operatorname{TPrA}]\left[\mathrm{Mn}(\mathrm{dca})_{3}\right]$ more closely resembled that of a type I isotherm-indicative of a microporous material such as a MOF. This may be due to the greater extent of surface porosity visible in $a_{\mathrm{g}}[\mathrm{TPrA}]\left[\mathrm{Mn}(\mathrm{dca})_{3}\right]$ when compared to $a_{\mathrm{g}}[\mathrm{TPrA}]\left[\mathrm{Co}(\mathrm{dca})_{3}\right]$. The significant increase in gas uptake in the glass forms of the HOIPs when compared to their crystalline counterparts is consistent with a large change
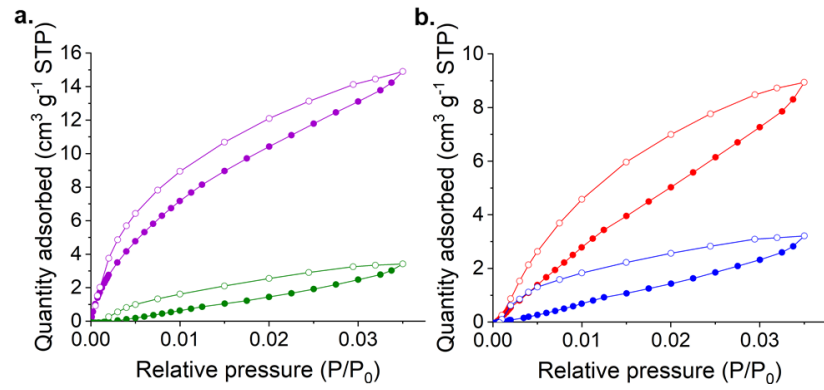

Figure 3. Carbon dioxide adsorption isotherms recorded for (a) [TPrA] $\left[\mathrm{Mn}(\mathrm{dca})_{3}\right]$ (green trace) and $a_{5}[\mathrm{TPrA}]\left[\mathrm{Mn}(\mathrm{dca})_{3}\right]$ (purple trace), (b) represent adsorption points and $a_{\mathrm{g}}[\mathrm{TP}$ circles represent deserption points. 
in structure, for example the previously proposed movement of the TPrA cation upon melting.

In this work, we have shown the mechanochemical synthesis of the HOIPs [TPrA][Mn(dca $\left.)_{3}\right]$ and [TPrA][Co(dca $\left.)_{3}\right]$ and the subsequent formation of bulk samples of their glass forms. Mechanochemical synthesis was used as an alternative to traditional slow-crystallisation and offered a green, rapid and scalable synthetic route to dicyanamide HOIPs of the form [TPrA] $\left[\mathrm{M}(\mathrm{dca})_{3}\right]$. Bulk samples of $a_{\mathrm{g}}[\operatorname{TPrA}]\left[\mathrm{Mn}(\mathrm{dca})_{3}\right]$ and $a_{\mathrm{g}}[\mathrm{TPrA}]\left[\mathrm{Co}(\mathrm{dca})_{3}\right]$ were formed in a standard tube furnace and the glasses were stable to all except strongly basic aqueous solutions. $\quad a_{\mathrm{g}}[\mathrm{TPr}]\left[\mathrm{Co}(\mathrm{dca})_{3}\right]$ displayed moderate hydrophilicity, with a mean water contact angle of $57.53 \pm 1.38^{\circ}$. The $\mathrm{CO}_{2}$ values of the HOIP glasses were higher than those of the corresponding non-porous crystalline samples, suggesting a significant structural change upon glass formation, such as the movement of A-site TPrA cations suggested in prior literature. Given the promising thermoelectric properties previously investigated for the glasses, the scalable synthesis of the materials may promote their applicability in real-world applications.

This work was financially supported by a Royal Society URF (UF150021) and research grant (RSG\R1\180395), a Philip Leverhulme Prize (2019) and the "Research Cooperability" Program of the Croatian Science Foundation funded by the European Union from the European Social Fund under the Operational Programme Efficient Human Resources 2014-2020, through grant PZS-2019-02-4129. In-situ PXRD was performed at the Deutsches Elektronen-Synchrotron PETRA III beamline P02.1. We wish to thank Dr. Ivana Brekalo for assistance with data collection and Dr. Stipe Lukin for help in data analysis.

\section{Conflicts of interest}

There are no conflicts to declare.

\section{Notes and references}

1

P. Jain, V. Ramachandran, R. J. Clark, H. D. Zhou, B. H. Toby, N. S. Dalal, H. W. Kroto and A. K. Cheetham, J. Am. Chem. Soc., 2009, 131, 13625-13627.

7 Y. Wu, S. Shaker, F. Brivio, R. Murugavel, P. D. Bristowe and A. K. Cheetham, J. Am. Chem. Soc., 2017, 139, 1699917002.

J. Snaith, Science, 2012, 338, 643-647.

M. Richter, L. Yang, L. Dai, M. Alsari, X. J. She, L. Liang, J.

Zhang, S. Lilliu, P. Gao, H. J. Snaith, J. Wang, N. C.

Greenham, R. H. Friend and D. Di, Nat. Photonics, 2018, 12, 783-789.
4 | J. Name., 2012, 00, 1-3
J. M. Bermúdez-García, M. Sánchez-Andújar, S. Yáñez-Vilar, S. Castro-García, R. Artiaga, J. López-Beceiro, L. Botana, Á. Alegría and M. A. Señarís-Rodríguez, Inorg. Chem., 2015, 54, 11680-11687.

J. M. Bermúdez-García, M. Sánchez-Andújar, S. Yáñez-Vilar, S. Castro-García, R. Artiaga, J. López-Beceiro, L. Botana, A. Alegría and M. A. Señarís-Rodríguez, J. Mater. Chem. $C$, 2016, 4, 4889-4898.

D. B. Mitzi, Chem. Mater., 1996, 8, 791-800.

T. Li, W. A. Dunlap-Shohl, E. W. Reinheimer, P. Le Magueres and D. B. Mitzi, Chem. Sci., 2019, 10, 1168-1175. B. K. Shaw, A. R. Hughes, M. Ducamp, S. Moss, A. Debnath, A. F. Sapnik, M. F. Thorne, L. N. McHugh, A. Pugliese, D. S. Keeble, P. Chater, J. M. Bermudez-Garcia, X. Moya, S. K. Saha, D. A. Keen, F. X. Coudert, F. Blanc and T. D. Bennett, Nat. Chem., 2021, 13, 778-785.

T. D. Bennett, Y. Yue, P. Li, A. Qiao, H. Tao, N. G. Greaves, T. Richards, G. I. Lampronti, S. A. T. Redfern, F. Blanc, O. K. Farha, J. T. Hupp, A. K. Cheetham and D. A. Keen, J. Am. Chem. Soc., 2016, 138, 3484-3492.

A. Qiao, H. Tao, M. P. Carson, S. W. Aldrich, L. M. Thirion, T. D. Bennett, J. C. Mauro and Y. Yue, Opt. Lett., 2019, 44, 1623.

S. Li, R. Limbach, L. Longley, A. A. Shirzadi, J. C. Walmsley, D. N. Johnstone, P. A. Midgley, L. Wondraczek and T. D. Bennett, J. Am. Chem. Soc., 2019, 141, 1027-1034. Y. Wang, H. Jin, Q. Ma, K. Mo, H. Mao, A. Feldhoff, X. Cao, Y. Li, F. Pan and Z. Jiang, Angew. Chemie - Int. Ed., 2020, 59, 4365-4369.

G. Jiang, C. Qu, F. Xu, E. Zhang, Q. Lu, X. Cai, S. Hausdorf, H. Wang and S. Kaskel, Adv. Funct. Mater., 2021, 31, 2104300. M. F. Thorne, M. L. R. Gómez, A. M. Bumstead, S. Li and T. D. Bennett, Green Chem., 2020, 22, 2505-2512. T. Stolar and K. Užarević, CrystEng Comm, 2020, 22, 45114525.

D. Prochowicz, M. Franckevičius, A. M. Cies̈lak, S. M. Zakeeruddin, M. Grätzel and J. Lewiński, J. Mater. Chem. A, 2015, 3, 20772-20777.

D. Prochowicz, P. Yadav, M. Saliba, M. Saski, S. M. Zakeeruddin, J. Lewiński and M. Grätzel, Sustain. Energy Fuels, 2017, 1, 689-693.

K. Užarević, I. Halasz and T. Friščić, J. Phys. Chem. Lett., 2015, 6, 4129-4140.

D. Crawford, J. Casaban, R. Haydon, N. Giri, T. McNally and S. L. James, Chem. Sci., 2015, 6, 1645-1649.

J. Sun and S. L. Simon, Thermochim. Acta, 2007, 463, 3240.

B. Karmakar, Fundamentals of Glass and Glass

Nanocomposites, Elsevier Inc., 2016.

A. Paul, J. Mater. Sci., 1977, 12, 2246-2268. S. Li, S. Yu, S. M. Collins, D. N. Johnstone, C. W. Ashling, A. F. Sapnik, P. A. Chater, D. S. Keeble, L. N. McHugh, P. A. Midgley, D. A. Keen and T. D. Bennett, Chem. Sci., 2020, 11, 9910-9918.

K. A. Cychosz and M. Thommes, Engineering, 2018, 4, 559566. 